\title{
Review Article \\ Recent Developments of Reflectarray Antennas in Dual-Reflector Configurations
}

\author{
Carolina Tienda, ${ }^{1}$ Manuel Arrebola, ${ }^{2}$ and José A. Encinar ${ }^{1}$ \\ ${ }^{1}$ Department of Electromagnetism and Circuit Theory, Technical University of Madrid, 30 Complutense Avenue, \\ 28040 Madrid, Spain \\ ${ }^{2}$ Department of Electrical Engineering, Group of Signal Theory and Communications, University of Oviedo, \\ Academic Campus, 33203 Gijón (Asturias), Spain \\ Correspondence should be addressed to Carolina Tienda, carotienda@gmail.com
}

Received 2 March 2012; Accepted 23 May 2012

Academic Editor: Raphaël Gillard

Copyright (C) 2012 Carolina Tienda et al. This is an open access article distributed under the Creative Commons Attribution License, which permits unrestricted use, distribution, and reproduction in any medium, provided the original work is properly cited.

Recent work on dual-reflector antennas involving reflectarrays is reviewed in this paper. Both dual-reflector antenna with a reflectarray subreflector and dual-reflectarrays antennas with flat or parabolic main reflectarray are considered. First, a general analysis technique for these two configurations is described. Second, results for beam scanning and contoured-beam applications in different frequency bands are shown and discussed. The performance and capabilities of these antennas are shown by describing some practical design cases for radar, satellite communications, and direct broadcast satellite (DBS) applications.

\section{Introduction}

Dual-reflector antennas are used to reduce the crosspolarization and the overall antenna volume in many applications, particularly in space applications. The use of a reflectarray as sub- or main reflector in a dual-reflector configuration [1] can provide some advantages in the antenna performance and in the manufacturing process. For example, a parabolic reflector with a reflectarray as subreflector has been proposed for compensating the errors on the surface of very large deployable reflectors $[2,3]$, for beam scanning in a limited angular range [4] and contoured beam applications [5]. This antenna configuration, based on parabolic main reflector and a reflectarray subreflector, offers several advantages compared to a single reflectarray antenna. First of all, it can provide a broad-band performance for large apertures, by using a reflectarray subreflector of small electrical dimensions and a large parabolic reflector. In addition, this dual-reflector configuration can be used to scan or reconfigure the beam by electronically controlling the phaseshift at the elements of a limited-size subreflectarray [4]. These configurations can be used for active or reconfigurable antennas by inserting amplifier modules or phase shifters on the elements of the reflectarray subreflector, in order to achieve high-power transmission or fine beam-pointing adjustment [6].

If the main reflector is replaced by a flat reflectarray, a dual-reflectarray antenna is obtained $[7,8]$. This configuration provides phase control on both surfaces, which can be used for different proposals as amplitude and phase synthesis [9], or to reduce the crosspolar radiation and shape the beam. The two-surface control permits to improve the performance of the antenna and extend the applicability of reflectarrays. Moreover this configuration provides advantages for transportable antenna application since the two flat arrays can be easily folded and deployed [10]. In a dualreflectarray antenna, active control devices can be included in both reflectarrays, being the subreflectarray preferred because of their smaller size. Finally, a dual-reflectarray antenna comprising a parabolic main reflectarray can be used to provide beam shaping in a broad band [11].

These antenna configurations including a controllable reflectarray subreflector can present a great potential in several space applications, such as Synthetic Aperture Radar (SAR), remote sensing, and reconfigurable antennas for direct broadcast satellites (DBSs). Although an SAR antenna 


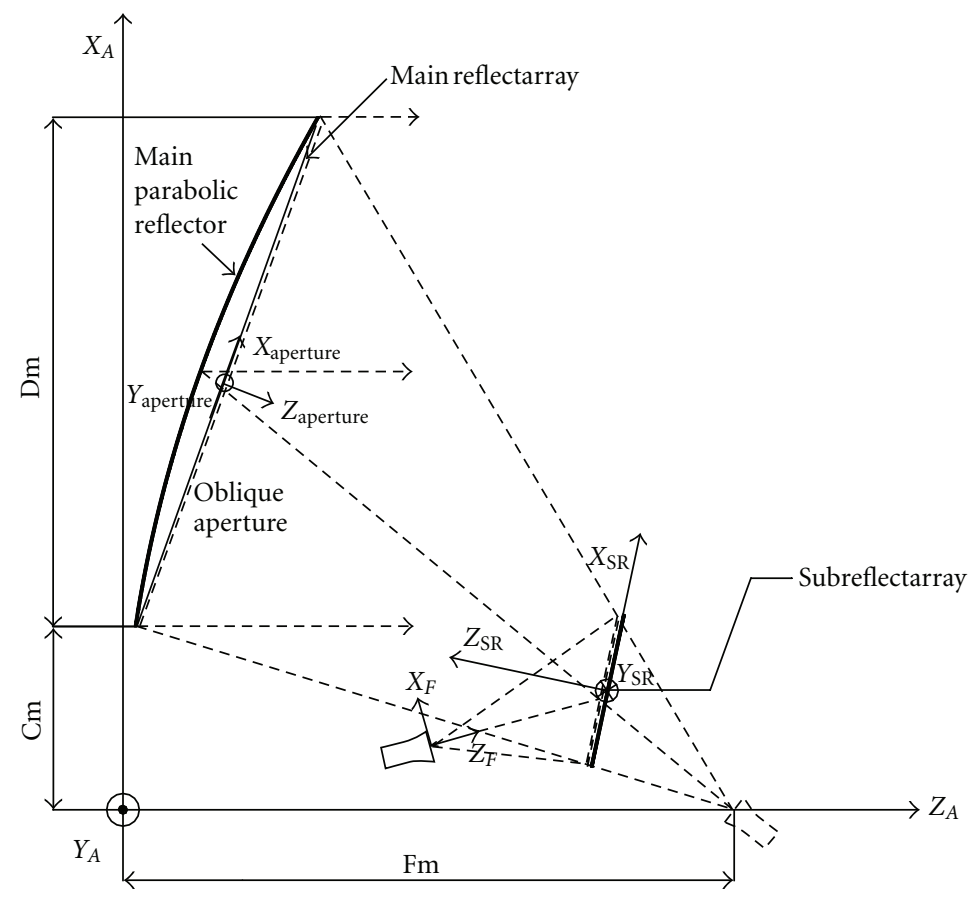

FIGURE 1: Scheme of dual-reflector antenna with a reflectarray subreflector and with a parabolic main reflector or a flat reflectarray as main reflector.

is usually implemented by active arrays, a reconfigurable reflectarray subreflector can provide beam scanning at a moderate cost and complexity. A second application is in radiometric remote sensing in millimeter and sub-millimeter frequency bands, which requires a beam scanning in a limited range actually implemented by motors [12]. An alternative for electronic beam scanning in this frequency bands can be reflectarrays based on liquid crystals [1315]. Finally, the reflectarray subreflector can be used to shape the beam in contoured-beam DBS antennas. In this configuration, the beam can be shaped by adjusting the phase-shift distribution on the subreflectarray, while the main parabolic reflector focuses the beam without any bandwidth limitation. If the phase is electronically controlled on the subreflectarray by using MEMS $[16,17]$ or varactor diodes [18], the beam can be reconfigured to change the coverage during the life-time of the satellite. This aspect is a real need in telecommunications and DBS missions, since the demand in data traffic (TV broadcast, phone, video or data transmission, multimedia direct-to-home, etc....) can change during the 15-20 year lifetime of the satellites.

\section{Analysis of Dual-Reflector Antennas Involving Reflectarrays}

As shown in Figure 1, the proposed antenna configuration is made up of three components: a primary feed, a flat subreflectarray, and a main reflector that can be either a parabolic classic reflector or a flat or curved reflectarray. Figure 1 shows a dual-offset optics using a horn antenna as primary feed, however a centered configuration can also be analysed. In the analysis of the dual-reflector antenna involving one or two reflectarrays, four coordinate systems are considered. Two of these systems are associated to two components of the antenna: the feed reference system $\left(X_{F}, Y_{F}, Z_{F}\right)$, which is centered at the phase centre of the primary feed; and the subreflectarray reference system, defined by $\left(X_{\mathrm{SR}}, Y_{\mathrm{SR}}, Z_{\mathrm{SR}}\right)$, whose origin is placed at the centre of the subreflectarray surface. The third coordinate system is used to define the aperture for the computation of the radiated field: the aperture reference system $\left(X_{\text {aperture }}, Y_{\text {aperture }}, Z_{\text {aperture }}\right)$, which is placed at the centre of the antenna aperture. All these coordinate systems are referred to as a common one, the antenna coordinate system $\left(X_{A}, Y_{A}, Z_{A}\right)$, with the $Z_{A}$ axis on the direction of the beam, as usual in reflector antennas.

This antenna can be analyzed through a modular procedure in four steps. First, the field radiated by the primary feed horn should be evaluated and the incident field must be computed at the centre of each subreflectarray cell. Although, in some cases, a conventional $\cos ^{q}(\theta)$ function can be used to model the field radiated by the feed within a good accuracy [19]. In general, the subreflectarray elements are placed in the Fresnel zone of the primary feed, and therefore the near field of the feed must be considered, which can be obtained through full-wave simulations, measurements of the feed or spherical mode expansion of the field radiated by the feed horn, $[20,21]$. Dual linear polarization is considered, vertical and horizontal, which corresponds to $X_{F}$ and $Y_{F}$ directions, respectively, according to Figure 1.

In the second step, the subreflectarray is analysed to compute the field reflected by each reflectarray cell. This analysis is carried out element by element through the general technique implemented for multilayer reflectarrays 


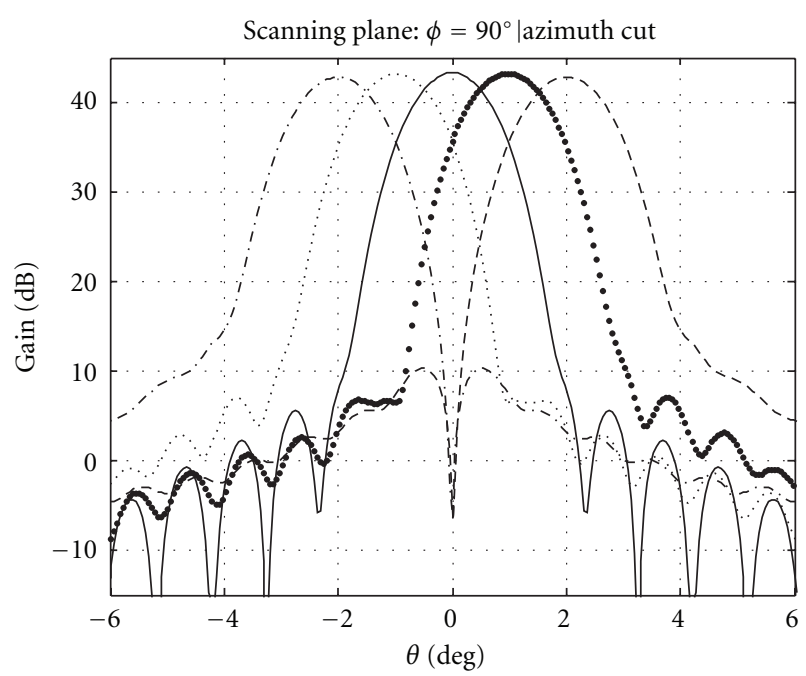

(a)

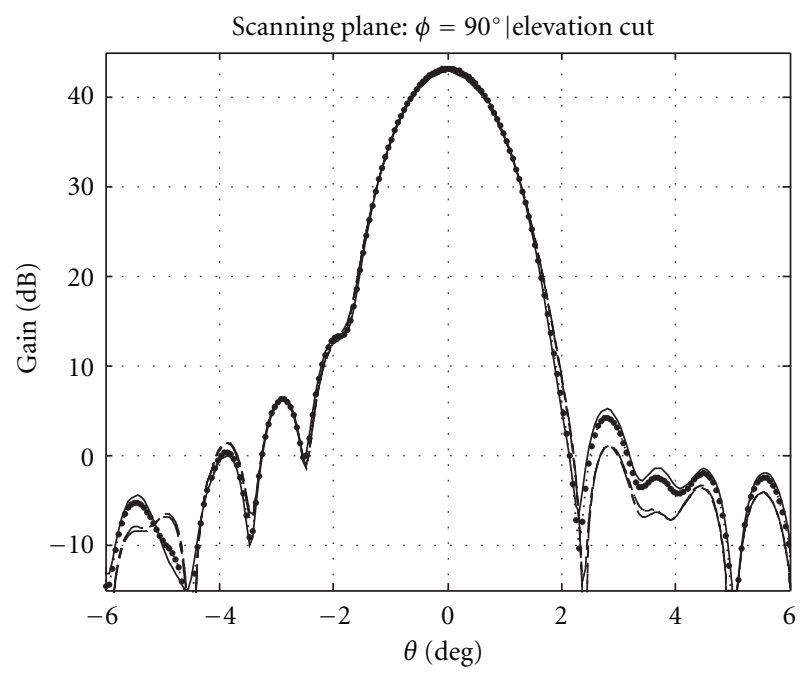

(b)

FIGURE 2: Simulated patterns of the dual-reflector antenna at $11.95 \mathrm{GHz}$ for beam scanning in azimuth in the principal planes. (a) Azimuth plane, (b) elevation plane.

[21-23]. The technique is based on Method of Moments in Spectral Domain, considering local periodicity and the real angle of incidence on each periodic cell of the wave coming from the feed, which has been demonstrated to be accurate for reflectarrays in single-reflector configurations. All the field components are taken into account, and the incident and reflected fields on each reflectarray cell, defined by the subindexes $(p, q)$, are related through a reflection matrix $\mathbf{P}^{p, q}$ as follows:

$$
E_{\mathrm{ref}}(p, q)=\mathbf{p}^{p, q} \cdot E_{\mathrm{in}}(p, q)
$$

The matrix $\mathbf{P}^{p, q}$ depends on the incidence angle and its complex elements relate the Cartesian components of the incident and the reflected fields, considering the crosspolarization produced by printed elements:

$$
\mathbf{P}^{p, q}=\left(\begin{array}{cc}
\rho_{x x}^{p, q} & \rho_{y x}^{p, q} \\
p, q & p, q \\
\rho_{x y} & \rho_{y y}^{p}
\end{array}\right) .
$$

Third, the analysis of the main reflector is carried out. In case of considering a classic parabolic reflector as main, the analysis will be based on physical optics. Thus, the electric printed currents on the reflector surface are computed, adding the contributions of the field radiated by all the subreflectarray elements. Once the currents are computed, the field is projected on the aperture plane through the Jacobian transformation.

In the case of having a flat reflectarray main reflector, it is analyzed using the same technique as for the subreflectarray, but we have to compute first the incident field on the elements of the main reflectarray. Now, the cells of the main reflectarray are placed in the Fresnel zone of the subreflectarray, but in the far-field region of the subreflectarray cells which are small apertures. Thus, the computation of the incident electric field on the main reflectarray surface can be computed as the superposition of all the contributions from the subreflector elements. In this case, the reflectarray surface is considered as the aperture plane. In the case that the main reflectarray is printed on a curved surface, the same procedure is applied but using a local coordinate system on each element of the main curved reflectarray and analyzing each element, assuming a locally flat periodic environment.

In the fourth step the radiation pattern of the entire antenna is computed starting from the electric field on the antenna aperture (aperture of the main reflector), using an algorithm based in FFT. Because of the analysis method, the radiation patterns are computed taking into account the ohmic losses in the reflectarray, the illumination, and spillover efficiencies.

\section{Applications}

Dual-reflector antennas involving reflectarrays have been identified as possible solutions for different applications. First, they can be used in beam-scanning applications since the beam pointing can be achieved with a small electronically-reconfigurable subreflectarray. In this applications, the use of a reflectarray as main reflector can be used to improve the folding and deploying performance of the antenna, which is very convenient for transportable antennas. Second, a reflectarray subreflector can be used to synthesize a contoured beam for DBS applications. These beams can also be achieved with a dual-reflectarray antenna using a main parabolic reflectarray to appropriately shape the beam in a broad frequency band and a passive subreflectarray, which can be used to improve the cross-polarization of the antenna. Finally, a reflectarray subreflector has been proposed to compensate the phase error introduced by main parabolic reflector surface because of the manufacturing tolerances [2]. 


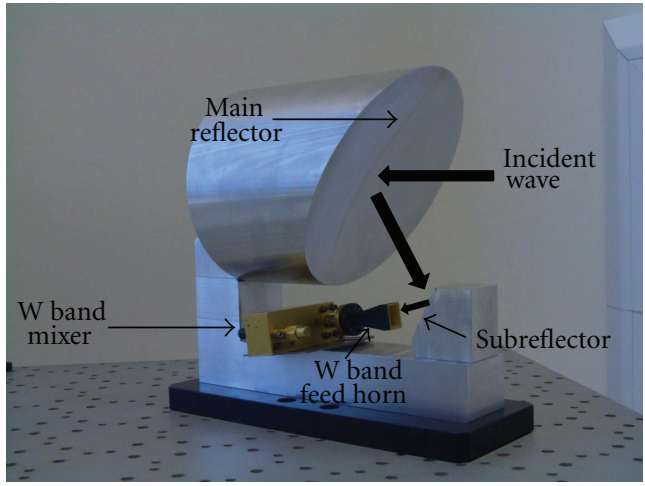

(a)

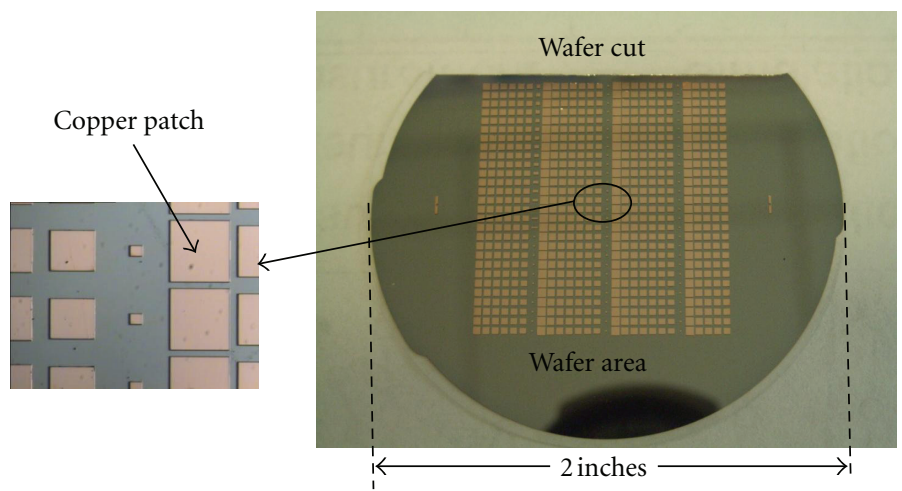

(b)

Figure 3: Dual reflector antenna at 94 GHz. (a) Photograph and (b) reflectarray subreflector. (From [21] () 2009 IEEE).

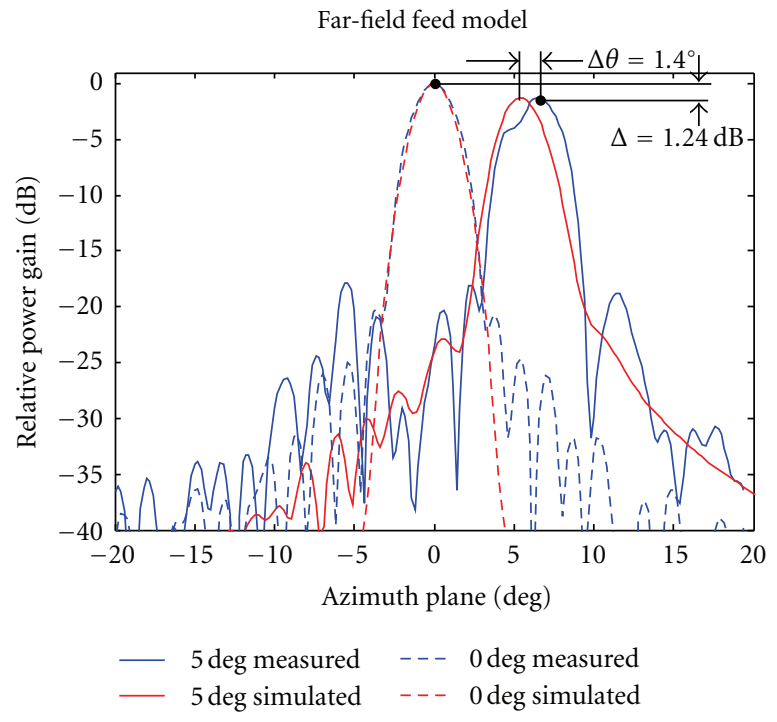

(a)

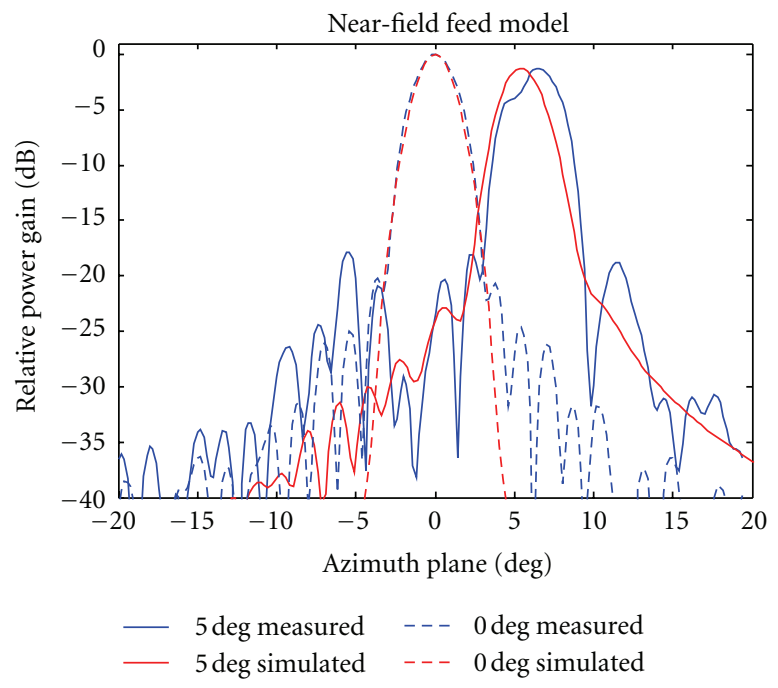

(b)

Figure 4: Measured and simulated radiation patterns of the dual reflector antenna with reflectarray subreflector in the azimuth plane at $94 \mathrm{GHz}$, using (a) far-field and (b) near-field models of the feed horn. (From [21] (c) 2009 IEEE).

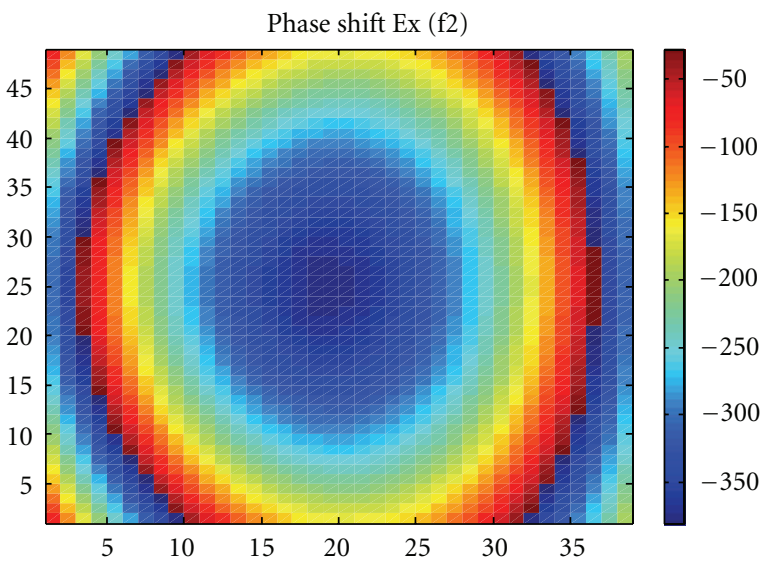

(a)

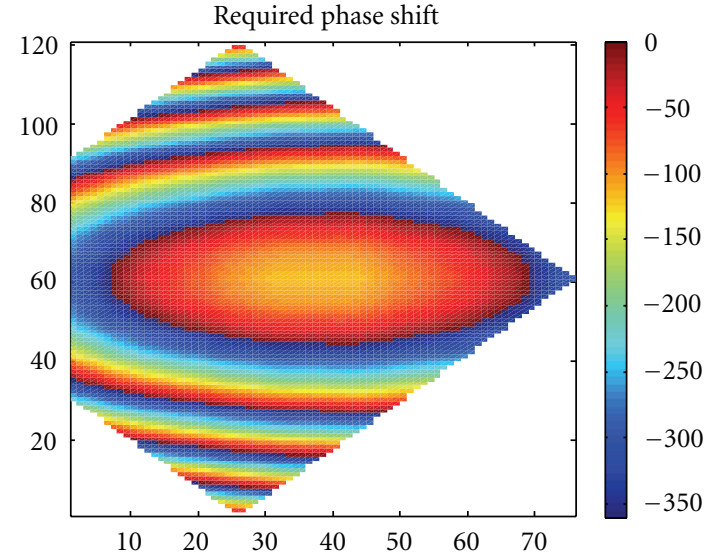

(b)

FIGURE 5: Phase distribution to be implemented on the subreflectarray (a) and on the main reflectarray at $12.75 \mathrm{GHz}$ (b). 


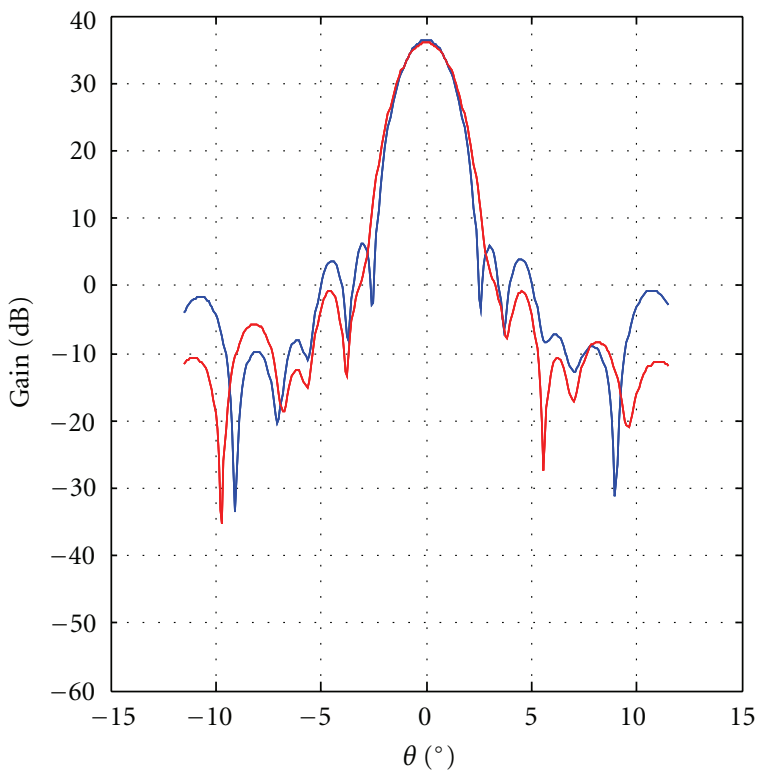

(a)

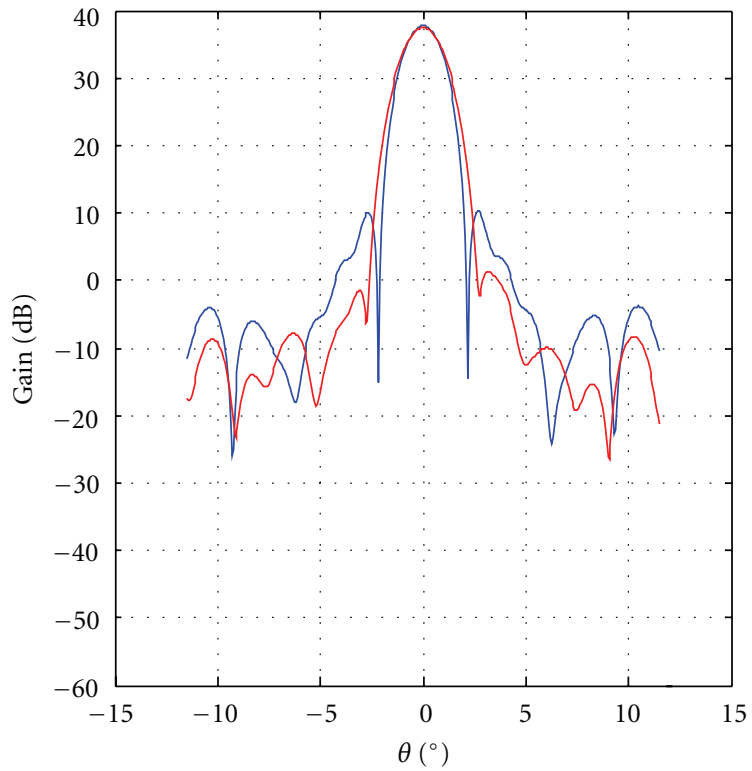

(b)

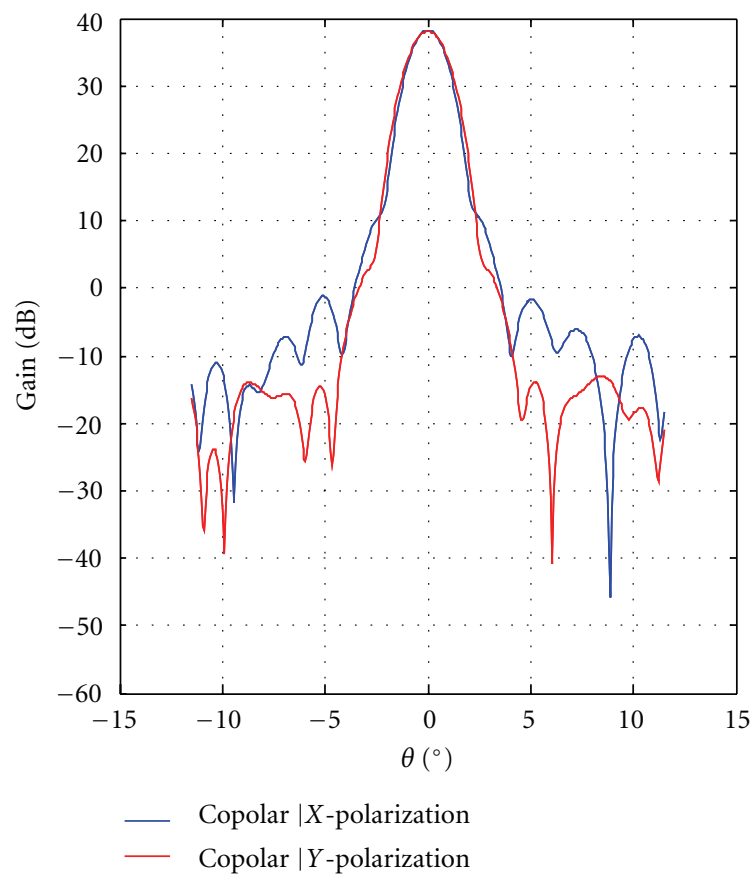

(c)

Figure 6: Azimuth cut of radiation pattern for $X$-polarisation at $10.7 \mathrm{GHz}$ (a), $12.75 \mathrm{GHz}$ (b), and $14.25 \mathrm{GHz}$ (c) when using the required phase distribution on the main reflectarray.

\subsection{Beam-Scanning Applications}

3.1.1. Dual-Reflector Antenna with Reflectarray Subreflector. The dual-reflector antenna with a reflectarray subreflector has been proposed as a high-gain beam-scanning antenna by adding controllable phase shifter on the subreflectarray. Thus, the beam pointing can be controlled through a reduced number of controls. In [4], the beam scanning performance of an antenna made of a $1.5 \mathrm{~m}$ aperture parabolic reflector and a rectangular subreflectarray $(520 \mathrm{~mm} \times 494 \mathrm{~mm})$ at $11.95 \mathrm{GHz}$ was studied in an angular range of $\pm 2^{\circ}$ from the antenna boresight. In this example, the feed horn is modeled as a $\cos ^{q}(\theta)$ function, with the $q$-factor chosen to provide an illumination taper on the subreflectarray around $-18 \mathrm{~dB}$, from the centre to the edges. The phase center of the horn is placed at coordinates $(-194,0,326) \mathrm{mm}$ referring to the subreflectarray coordinate system $\left(X_{R}, Y_{R}, Z_{R}\right)$, and the center of the subreflectarray is placed at coordinates $(294,0,1174) \mathrm{mm}$ 


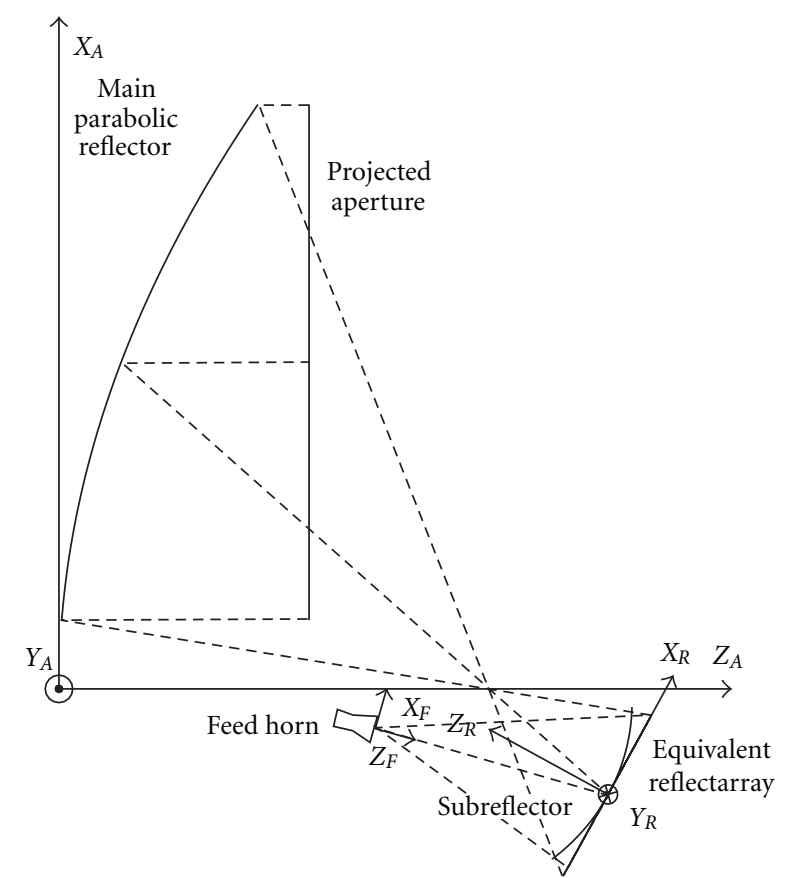

Figure 7: Scheme of the subreflectarray-parabolic reflector antenna.

in the antenna reference system $\left(X_{A}, Y_{A}, Z_{A}\right)$. In this work, the scanning is achieved by introducing a progressive phase shift along the reflectarray subreflector. This approach permits to deflect the beam in one plane. For example, if a progressive phase is introduced along the $Y_{R}$-axis of the subreflectarray, the beam is scanned the azimuth plane $\left(Y_{A} Z_{A}\right)$. As a result, the control circuits are simplified, since the phase control should be the same for all the elements in a column or a row. The radiation patterns of this antenna for the $X_{A}$ polarization (electric field in $X_{A}$ direction) in the principal planes are shown in Figure 2, assuming an ideal progressive phase on the subreflectarray for beam scanning in azimuth. The gain has been computed for a beam scanning from $-2^{\circ}$ to $+2^{\circ}$, showing a reduction in gain less than $1 \mathrm{~dB}$ in all the cases. In the case of ideal phase shifters considered here, the cross-polarization for the overall antenna is slightly increased when the beam is defocused, being in the order of $-30 \mathrm{~dB}$ below the copolar gain. If a larger beam-scanning angular range is required, the phase control at row or column level is not accurate enough and a synthesis technique must be applied to obtain the phase distribution required on the subreflectarray surface for each beam pointing. A simple synthesis technique for this application is discussed in [24] showing that the angular scanning range can be extended up to $\pm 7^{\circ}$, with the beam distorted for broader angles because of the spillover.

3.1.2. $94 \mathrm{GHz}$ Dual-Reflector Antenna. The beam deflecting performance of this antenna configuration has been demonstrated in the $94 \mathrm{GHz}$ band, as discussed in [21]. In this work, the dual-reflector antenna is designed, manufactured, and tested to show that it is possible to steer the beam up to $\pm 5^{\circ}$ from boresight by replacing a flat metal subreflector with a small reflectarray. The antenna demonstrator is made up of an offset parabolic reflector of diameter $120 \mathrm{~mm}(38 \lambda)$ and a flat subreflector printed on a $50 \mathrm{~mm}$ diameter quartz wafer, see Figure 3. The beam scanning capability of the antenna is demonstrated by replacing a solid metal flat subreflector, which produces a focused beam in the boresight direction, by a passive $28 \times 28$ elements reflectarray printed on a $115 \mu \mathrm{m}$ thick metal-backed quartz wafer. The reflectarray is made of a single layer of varying-size patches, see Figure 3(b), designed to produce a progressive phase distribution in the $Y_{R}$ direction to deflect the beam $5^{\circ}$ in the azimuth plane. The copolar far field radiation patterns of the antenna were measured between $-20^{\circ}$ and $+20^{\circ}$ in the azimuth plane at $94 \mathrm{GHz}$ at Queen's University Belfast (QUB). Figure 4 shows the measured radiation pattern using the reflectarray subreflector to tilt the beam $5^{\circ}$ and also the results using a flat metallic subreflector to produce a boresight-directed beam. The measured radiation patterns are compared in Figure 4 with the simulated patterns obtained through the technique described in the previous section, in two cases: (a) considering the ideal far-field model and (b) considering the near field of the primary feed horn, see [21]. These results show very good agreement with the measurements when the near-field model is used, which demonstrates the need to use the near field radiated by the feed for an accurate analysis of the antenna. The gain for the boresight beam computed by the analysis tool is $38.7 \mathrm{dBi}$, with the gain reduced in $1.26 \mathrm{~dB}$ for the deflected beam. The predicted reduction in gain is $0.86 \mathrm{~dB}$ due to the $5^{\circ}$ beam deflection and $0.40 \mathrm{~dB}$ because of phase errors and losses in the reflectarray. Some distortion of the tilted beam and a deviation of $1.4^{\circ}$ in the beam direction are found because the antenna design was carried by implementing a progressive phase distribution on the reflectarray assuming the far-field model of the feed. However the far-field model of the horn does not provide a realistic estimation of the incident field, neither in amplitude nor in phase, as shown in [21]. In order to reduce the distortion of the deflected beam, the reflectarray subreflector should be designed considering the near field of the feed horn [20].

\subsubsection{Dual-Reflectarray Antenna for Bidirectional Satellite} Link. A dual-reflectarray antenna can be used for beamscanning application, [10]. This configuration is very convenient for transportable antennas since the two flat reflectarrays are ease to fold and deploy. In this case, the dualreflectarray antenna is made up of a passive sub- and main reflectarrays. The main reflectarray can be also implemented with 1-bit electronic control for beam pointing. The antenna is defined to provide a directive beam in receive $(10.70-12.75 \mathrm{GHz})$ and transmit $(14.0-14.5 \mathrm{GHz})$ frequency bands with electronic scanning capabilities within a limited angular range when using 1-bit electronic control elements on the main reflectarray. As discussed in [10], the main reflectarray has pentagonal shape with dimensions of 1500 $\mathrm{mm} \times 750 \mathrm{~mm}$. This reflectarray comprises 5250 elements distributed in 75 rows and 120 columns. The elements 


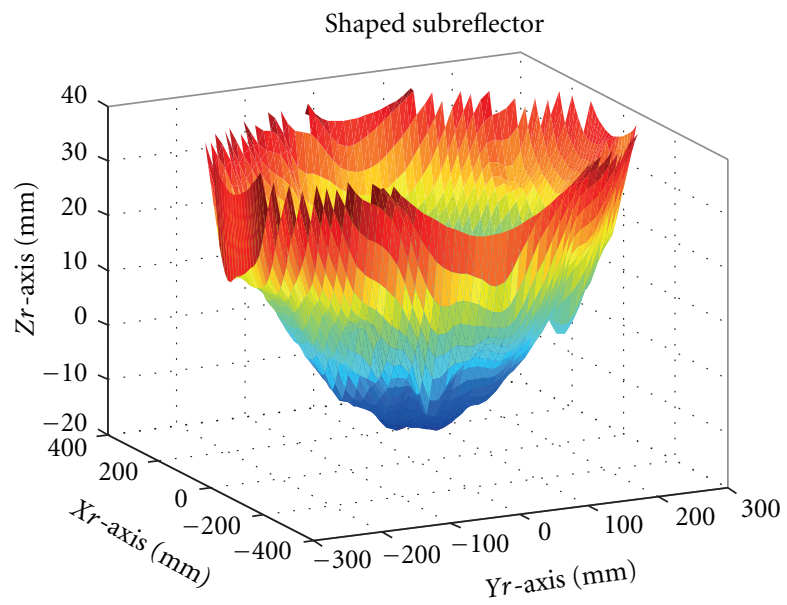

(a)

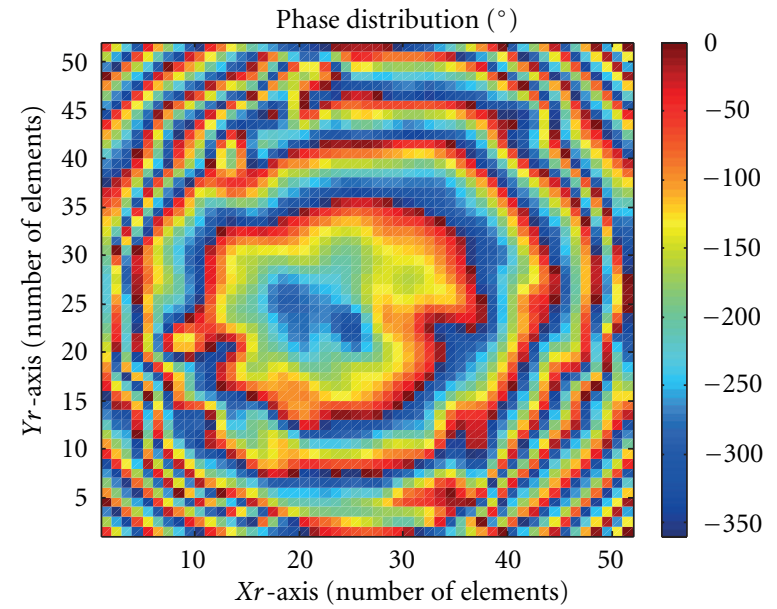

(b)

FIGURE 8: Shaped ellipsoid as subreflector (a) and phase distribution of the reflection coefficient for the equivalent reflectarray at $11.95 \mathrm{GHz}$ (b).

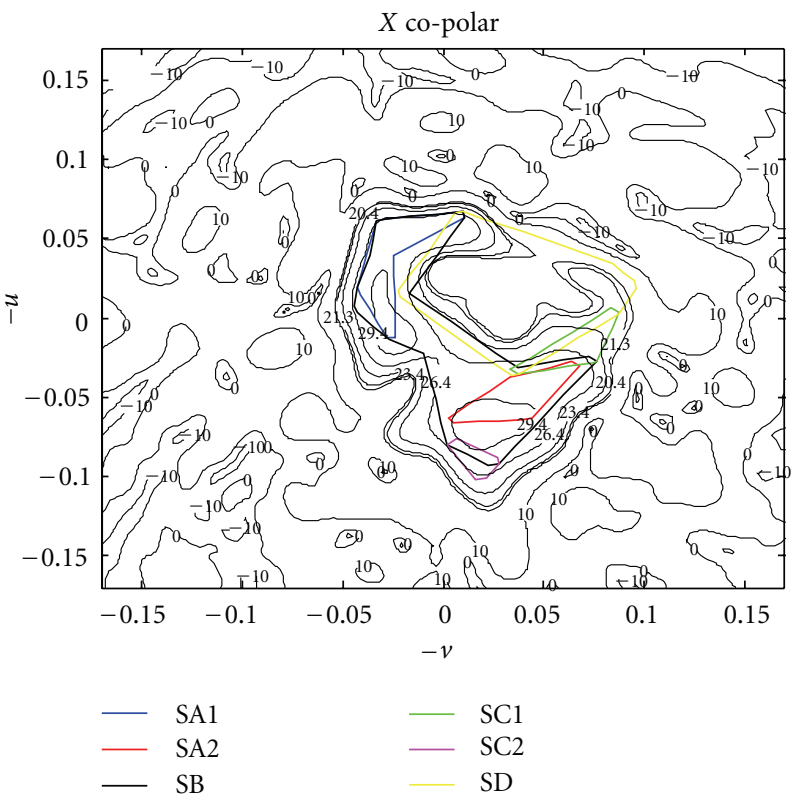

(a)

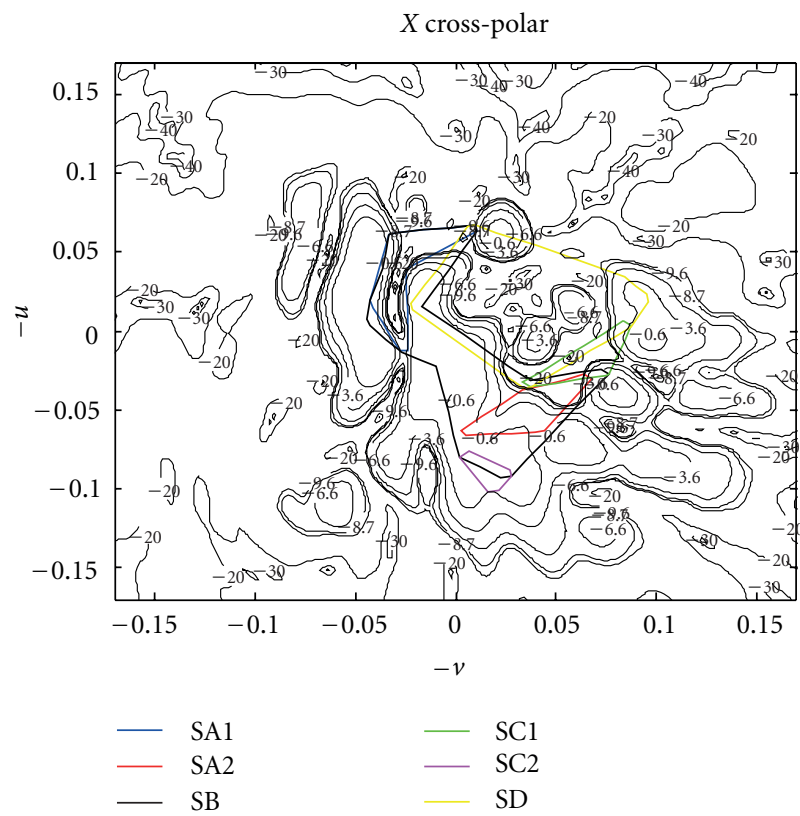

(b)

FIgURE 9: Copolar (a) and cross-polar (b) radiation patterns at $11.95 \mathrm{GHz}\left(X_{m}\right.$-pol.) with the coverage template superimposed.

are arranged in a periodic lattice of $10 \mathrm{~mm} \times 10 \mathrm{~mm}$. The subreflectarray is tilted $27.69^{\circ}$ with respect to the main reflectarray, with dimensions of $475 \mathrm{~mm} \times 600 \mathrm{~mm}$ and placed with its centre at coordinates $(-473,0.0,690) \mathrm{mm}$ referring to the main coordinate system. The subreflectarray is rectangular, with $38 \times 48$ elements in a periodic mesh of $12.5 \mathrm{~mm} \times 12.5 \mathrm{~mm}$ periodicity. The phase centre of the feed horn is located at coordinates $(300,0,30) \mathrm{mm}$ in the main reflectarray coordinate system. The subreflectarray has been designed to provide a very large equivalent focal distance in order to illuminate efficiently the main reflector. A simple model of the horn antenna based on $\cos ^{q}(\theta)$ function is used. The $q$ factor is defined at each frequency to provide an illumination level at the edge of the main reflector between $-10 \mathrm{~dB}$ and $-15 \mathrm{~dB}$.

Figure 5(a) shows the phase distribution on the subreflectarray. Figure 5(b) shows the required phase shift for the main reflectarray to obtain a pencil beam pointing at $40^{\circ}$ from the $z$-axis. The radiation patterns at $10.7 \mathrm{GHz}$, 12.75 GHz (Rx), and $14.25 \mathrm{GHz}(\mathrm{Tx})$ are shown in Figure 6. 


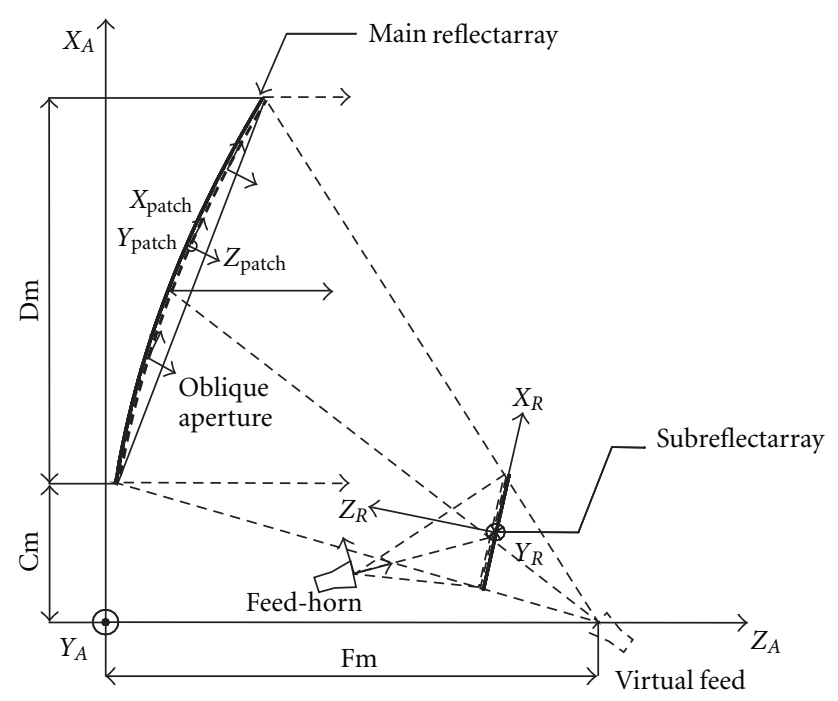

FIGURE 10: Scheme of a dual-reflectarray structure analysis.

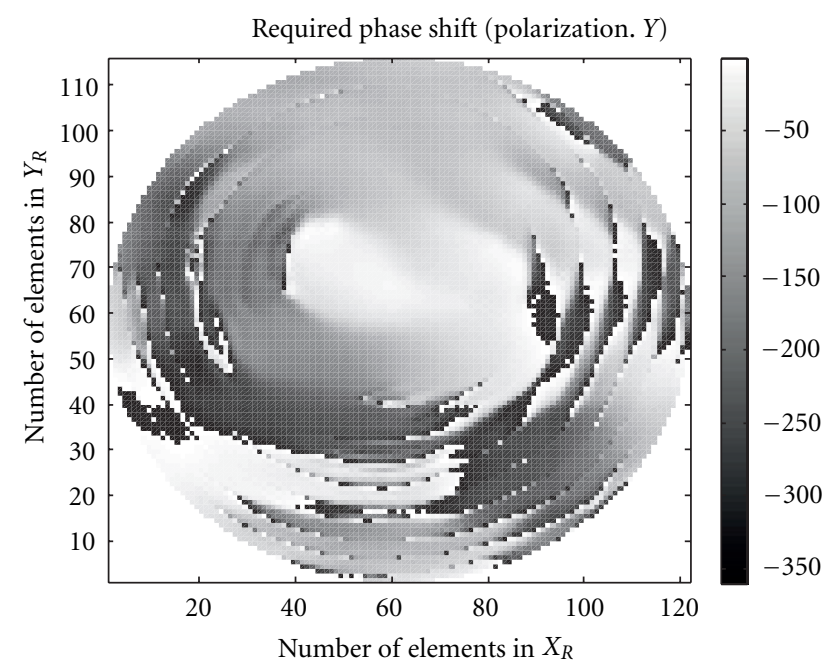

FIGURE 11: Required phase-shift for a $1.5 \mathrm{~m}$ parabolic reflectarray at $11.95 \mathrm{GHz}, F / D=1.2[25]$.

\subsection{Contoured-Beam Antenna for DBS Applications}

3.2.1. Contoured-Beam Antenna with a Shaped Subreflectarray. A dual-reflector antenna based on a reflectarray subreflector and a main parabolic reflector has been proposed to obtain contoured beams for DBS applications [5]. The classic solution in this application consists on single or dual reflector antenna based on shaped reflectors. This solution is expensive and time consuming since the reflector surface shaping depends on the coverage footprint, and the manufacture requires specific moulds which are different for each mission. In the case of a dual-reflector antenna with a reflectarray sub-reflector, the beam shaping can be achieved by the phase-shift introduced on the reflectarray surface. In this work, the coverage specified for a South American mission on Amazonas satellite in Ku-band transmit band $(11.95 \mathrm{GHz})$ is selected. The geometry of the antenna is derived from a dual-offset Gregorian optics with a main aperture of $1.5 \mathrm{~m}$ diameter, see Figure 7. In this antenna, the ellipsoidal subreflector $(0.5 \mathrm{~m}$ diameter $)$ is replaced by a reflectarray with the appropriate phasing. As shown in Figure 7 , the equivalent subreflectarray is placed on the $X_{R} Y_{R}$ plane, being its centre placed at $(-308,0,1596) \mathrm{mm}$ in the antenna coordinate system. The subreflectarray is made up of $51 \times 51$ elements arranged in regular mesh with period $11 \mathrm{~mm} \times 11 \mathrm{~mm}$. As in previous sections, the feed horn is modeled as a $\cos ^{q}(\theta)$ function which provides an illumination taper at edges of $-22 \mathrm{~dB}$ in order to reduce the spillover.

In order to synthesize the subreflectarray phases, the shape of a subreflector that fulfils the coverage requirements is achieved and used as the starting point in the process to obtain the phase distribution of the reflection coefficient of the equivalent reflectarray, see Figure $8(\mathrm{a})$. The phase distribution shown in Figure 8(b) is the same for the two linear polarizations of the antenna, since it has been derived from the distances from the shaped ellipsoid to the equivalent reflectarray plane. The radiation pattern for $X_{A}$ polarization of the antenna is plotted in Figure 9, being the results similar for the orthogonal polarization. Co- and cross-polar components have been calculated considering the elements of the reflectarray as ideal phase shifters and no losses (ideal reflectarray elements), see Figures 9(a) and 9(b). The shape of the footprint obtained shows some distortion. However, the performance is near to the coverage required mask; see Figure 9(a). This phase distribution can be a suitable starting point for a further optimization process.

\subsubsection{Parabolic Reflectarray in Dual-Reflector Configuration.} The South American coverage antenna shown in the previous section can also be implemented with a dual-reflectarray antenna using a main parabolic reflectarray and a flat subreflectarray. The contoured beam was previously synthesized in a single offset parabolic reflectarray as discussed in [25]. The general scheme of this antenna is shown in Figure 10.

The antenna projected aperture has a diameter of $1.5 \mathrm{~m}$, resulting an elliptic parabolic reflectarray of axes $1551 \mathrm{~mm} \times$ $1500 \mathrm{~mm}$. The clearance of the antenna is $200 \mathrm{~mm}$ and the focal distance is $1800 \mathrm{~mm}$. The subreflector is a flat reflectarray with axes $598 \mathrm{~mm} \times 559 \mathrm{~mm}$. In principle, the antenna optics is defined to compact the overall volume. In this case, the subreflectarray behaves as a metal plane introducing a constant phase distribution, equivalent to a flat metal plate, in order to be able to compare the results with those previously reported for a single offset parabolic reflectarray [25]. Although the degree of freedom in the phase distribution on the subreflectarray can be used to reduce the cross-polarization of the antenna. The phase distribution on the main reflectarray surface required to obtain the South American coverage is shown in Figure 11. To implement this phase distribution, a unit cell with two stacked rectangular patches has been used as a reflectarray element. The patches are arranged in a periodic lattice of $13 \mathrm{~mm} \times 13 \mathrm{~mm}$ and they are assumed to be printed on a 125 micron Kapton film bonded to a quartz-fabric composite 


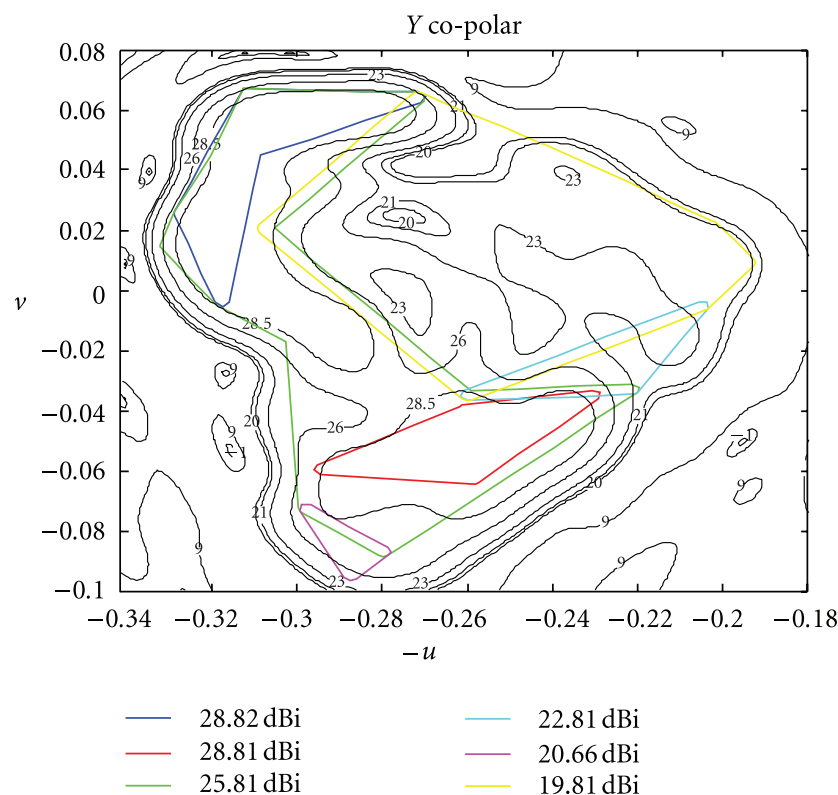

(a)

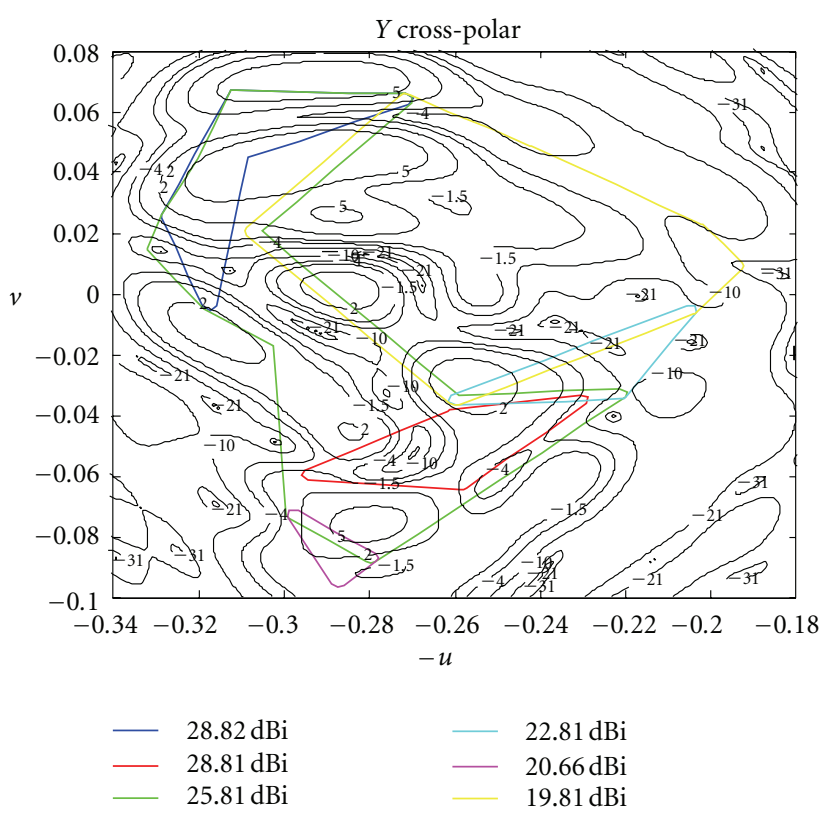

(b)

Figure 12: Radiation patterns obtained by MoM for a two-layer reflectarray in Tx band, 11.95 GHz. (a) Copolar component. (b) Crosspolar component.

layer and separated by quartz-honeycomb separators with $3.5 \mathrm{~mm}$ thickness.

The radiation pattern was computed at three frequencies: $11.7,11.95$, and $12.2 \mathrm{GHz}$, corresponding to the central and extreme frequencies of the transmit band (TX). Accurate predictions of both copolar and cross-polar radiation patterns at central frequency are provided in Figure 12, showing that the contoured patterns are close to the requirements, which are also shown in the figures as coloured polygons. Similar results were obtained for the extreme frequencies of the band. Two small holes at the border of the $28.81 \mathrm{dBi}$ contour are found as a result of ohmic losses and phase errors produced by the approximate design technique, in which the pattern synthesis is applied on the planar aperture and the required phase distribution is obtained by geometrical optics. The contoured patterns can be improved by a suitable pattern synthesis taking into account the curved surface.

The analysis technique provides an accurate prediction of the cross-polarization pattern performance, since it takes into account the cross-polarization produced by the printed patches and by the offset reflector. The cross-polarization shown in Figure 8(b) can be reduced by synthesizing a proper phase distribution on the subreflectarray surface.

\section{Conclusions}

A review of recent work on dual-reflector antennas involving reflectarrays has been presented, considering a flat reflectarray subreflector and parabolic reflector, flat reflectarray, or parabolic reflectarray as main reflectors. A modular technique for the analysis of dual-offset antennas involving one or two reflectarrays has been described. The technique has been applied to analyze several antenna configurations for beam scanning and contoured beam for DBS applications.

Three examples of beam scanning dual-reflector antennas involving reflectarrays have been presented. First, a highgain beam-scanning antenna based on a flat subreflectarray and a main parabolic reflector has been studied. The beam scanning can be realized by adding controllable phase shifters on the subreflectarray. The beam scanning performance of an antenna in a reduced angular range of $\pm 2^{\circ}$ has been presented. Second, a dual-offset antenna with a reflectarray sub-reflector working at $94 \mathrm{GHz}$ has been designed to provide electronic beam scanning in a limited angular range $\left( \pm 5^{\circ}\right)$. This antenna has been manufactured and tested, demonstrating the capability of this configuration for beam scanning in submillimeter-wave frequencies. The results show that a near-field model of the feed horn is necessary for an accurate analysis and design of the antenna. Third, a dual-reflectarray antenna with electronic beam steering has been presented for portable systems to provide $\mathrm{Ku}-$ band satellite links with automatic pointing capabilities. The antenna configuration comprises a passive subreflectarray and a main reflectarray with 1-bit electronic control to provide a pencil beam steerable in $\mathrm{a} \pm 5^{\circ}$ angular range.

Dual-reflector antennas with one or two reflectarrays have also been proposed for contoured-beam applications. Two antennas providing South American coverage have been presented. A first solution consists of a dual-reflector dualoffset antenna with a $56 \mathrm{~cm}$ subreflectarray and a $1.5 \mathrm{~m}$ main parabolic reflector. In this case the coverage is achieved by synthesizing the phase distribution on the subreflectarray surface. The same coverage is also obtained by a dualreflectarray antenna with $1.5 \mathrm{~m}$ parabolic reflectarray. In 
this antenna the beam shaping is achieved by the main reflectarray, while sub reflectarray can be used to reduce the cross-polar radiation of the entire antenna.

All the configurations and applications presented in the paper show the versatility that a dual-offset antenna involving reflectarrays may offer, as beam scanning, beam shaping, or reduction of cross-polarization.

\section{Acknowledgments}

This work has been supported in part by the "Ministerio de Ciencia e Innovación" (Spanish Government) under projects CONSOLIDER-INGENIO CSD2008-00068 (TERASENSE) and TEC2010-17567 and also by the European Space Agency (ESA).

\section{References}

[1] B. Khayatian, Y. Rahmat-Samii, and J. Huang, "Radiation characteristics of reflectarray antennas: methodology and applicatios to dual configurations," in Proceedings of the European Conference on Antennas and Propagation (EuCAP '06), Nice, France, November 2006.

[2] S. Xu, H. Rajagopalan, Y. Rahmat-Samii, and W. A. Imbriale, "A novel reflector surface distortion compensating technique using a sub-reflectarray," in Proceedings of the IEEE Antennas and Propagation Society International Symposium (AP-S '07), pp. 5315-5318, Honolulu, Hawaii, USA, June 2007.

[3] S. Xu, Y. Rahmat-Samii, and W. A. Imbriale, "Subreflectarrays for reflector surface distortion compensation," IEEE Transactions on Antennas and Propagation, vol. 57, no. 2, pp. 364-372, 2009.

[4] M. Arrebola, L. de Haro, and J. A. Encinar, "Analysis of dualreflector antennas with a reflectarray as subreflector," IEEE Antennas and Propagation Magazine, vol. 50, no. 6, pp. 39-51, 2008.

[5] M. Arrebola, L. De Haro, J. A. Encinar, and L. F. De La Fuente, "Contoured-beam Gregorian antenna with a reflectarray as subreflector," in Proceedings of the 2nd European Conference on Antennas and Propagation (EuCAP '07), Edinburgh, UK, November 2007.

[6] W. Huang and J. R. Lesh, "Reflectarray elements on subreflector for fine beam pointing," JPL New Technology Report 41562, 2004.

[7] W. Menzel and D. Kessler, "A folded reflectarray antenna for 2D scanning," in German Microwave Conference, pp. 1-14, March 2009.

[8] C. Tienda, M. Arrebola, J. A. Encinar, and G. Toso, "Analysis of a dual-reflect array antenna," in Proceedings of the IET on Microwaves, Antennas \& Propagation, vol. 5, no. 13, pp. 16361645, October 2011.

[9] R. Leberer and W. Menzel, "A dual planar reflectarray with synthesized phase and amplitude distribution," IEEE Transactions on Antennas and Propagation, vol. 53, no. 11, pp. 35343539, 2005.

[10] C. Tienda, J. A. Encinar, S. Montori, R. Vincenti Gatti, M. Arrebola, and R. Sorrentino, "Dual-reflectarray antenna for bidirectional satellite links in Ku-band," in Proceedings of the 5th European Conference on Antennas and Propagation (EUCAP '11), pp. 1404-1407, Rome, Italy, April 2011.
[11] Y. Álvarez, F. Las-Heras, and M. R. Pino, "Reconstruction of equivalent currents distribution over arbitrary threedimensional surfaces based on integral equation algorithms," IEEE Transactions on Antennas and Propagation, vol. 55, no. 12, pp. 3460-3468, 2007.

[12] R. J. Martin and D. H. Martin, "Quasi-optical antennas for radiometric remote-sensing," Electronics and Communication Engineering Journal, vol. 8, no. 1, pp. 37-48, 1996.

[13] A. Möessinger, R. Marin, S. Mueller, J. Freese, and R. Jakoby, "Electronically reconfigurable reflectarrays with nematic liquid crystals," Electronics Letters, vol. 42, no. 16, pp. 899-900, 2006.

[14] W. Hu, M. Y. Ismail, R. Cahill et al., "Liquid-crystal-based reflectarray antenna with electronically switchable monopulse patterns," Electronics Letters, vol. 43, no. 14, pp. 899-900, 2007.

[15] A. Moessinger, R. Marin, D. Eicher, R. Jakoby, and H. Schlaak, "Liquid crystal reflectarray with electronic 2D-reconfiguration capability," in Proceedings of the 29th ESA Antenna Workshop on Multiple Beams and Reconfigurable Antennas, pp. 67-70, April 2007.

[16] L. Marcaccioli, B. Mencagli, R. Vincenti Gatti et al., "Beam steering MEMS mm-wave reflectarrays," in MEMSWAVE International Symposium, Orvieto, Italy, June 2006.

[17] H. Rajagopalan, Y. Rahmat-Samii, and W. A. Imbriale, "RF MEMS actuated reconfigurable reflectarray patch-slot element," IEEE Transactions on Antennas and Propagation, vol. 56, no. 12, pp. 3689-3699, 2008.

[18] S. V. Hum, M. Okoniewski, and R. J. Davies, "Realizing an electronically tunable reflectarray using varactor diode-tuned elements," IEEE Microwave and Wireless Components Letters, vol. 15, no. 6, pp. 422-424, 2005.

[19] M. Arrebola, J. A. Encinar, and M. Barba, "Multifed printed reflectarray with three simultaneous shaped beams for LMDS central station antenna," IEEE Transactions on Antennas and Propagation, vol. 56, no. 6, pp. 1518-1527, 2008.

[20] M. Arrebola, Y. Álvarez, J. A. Encinar, and F. Las-Heras, "Accurate analysis of printed reflectarrays considering the near field of the primary feed," IET Microwaves, Antennas and Propagation, vol. 3, no. 2, pp. 187-194, 2009.

[21] W. Hu, M. Arrebola, R. Cahill et al., "94 GHz dual-reflector antenna with reflectarray subreflector," IEEE Transactions on Antennas and Propagation, vol. 57, no. 10, part 2, pp. 30433050, 2009.

[22] C. Wan and J. A. Encinar, "Efficient computation of generalized scattering matrix for analyzing multilayered periodic structures," IEEE Transactions on Antennas and Propagation, vol. 43, no. 11, pp. 1233-1242, 1995.

[23] J. A. Encinar, "Design of two-layer printed reflectarrays using patches of variable size," IEEE Transactions on Antennas and Propagation, vol. 49, no. 10, pp. 1403-1410, 2001.

[24] M. Arrebola, E. Carrasco, and J. A. Encinar, "Beam scanning antenna using a reflectarray as sub-reflector," ACES Journal, vol. 26, no. 6, 2011.

[25] J. A. Encinar, M. Arrebola, and G. Toso, "A parabolic reflectarray for a bandwidth improved contoured beam coverage," in Proceedings of the 2nd European Conference on Antennas and Propagation (EuCAP '07), EICC, November 2007. 

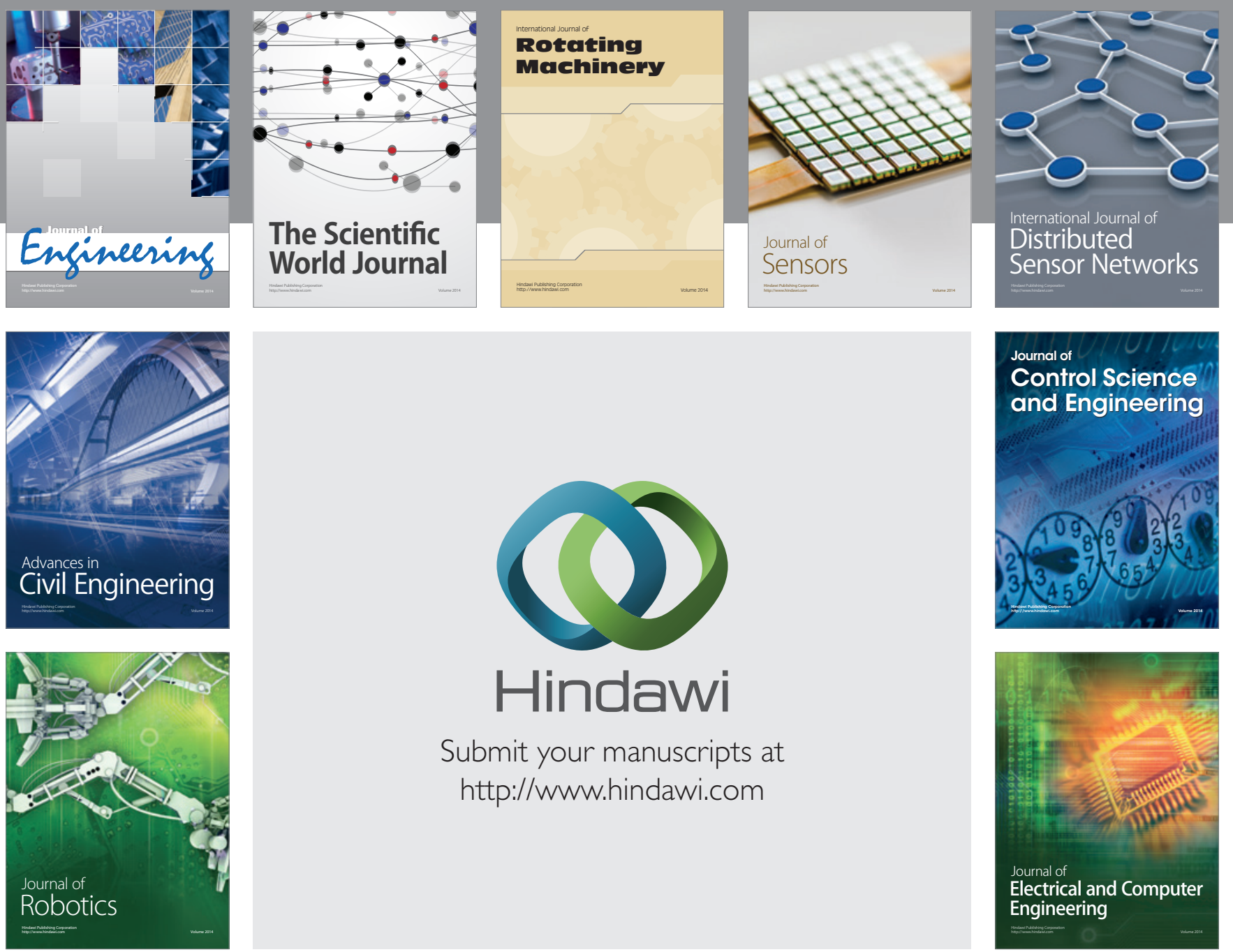

Submit your manuscripts at

http://www.hindawi.com
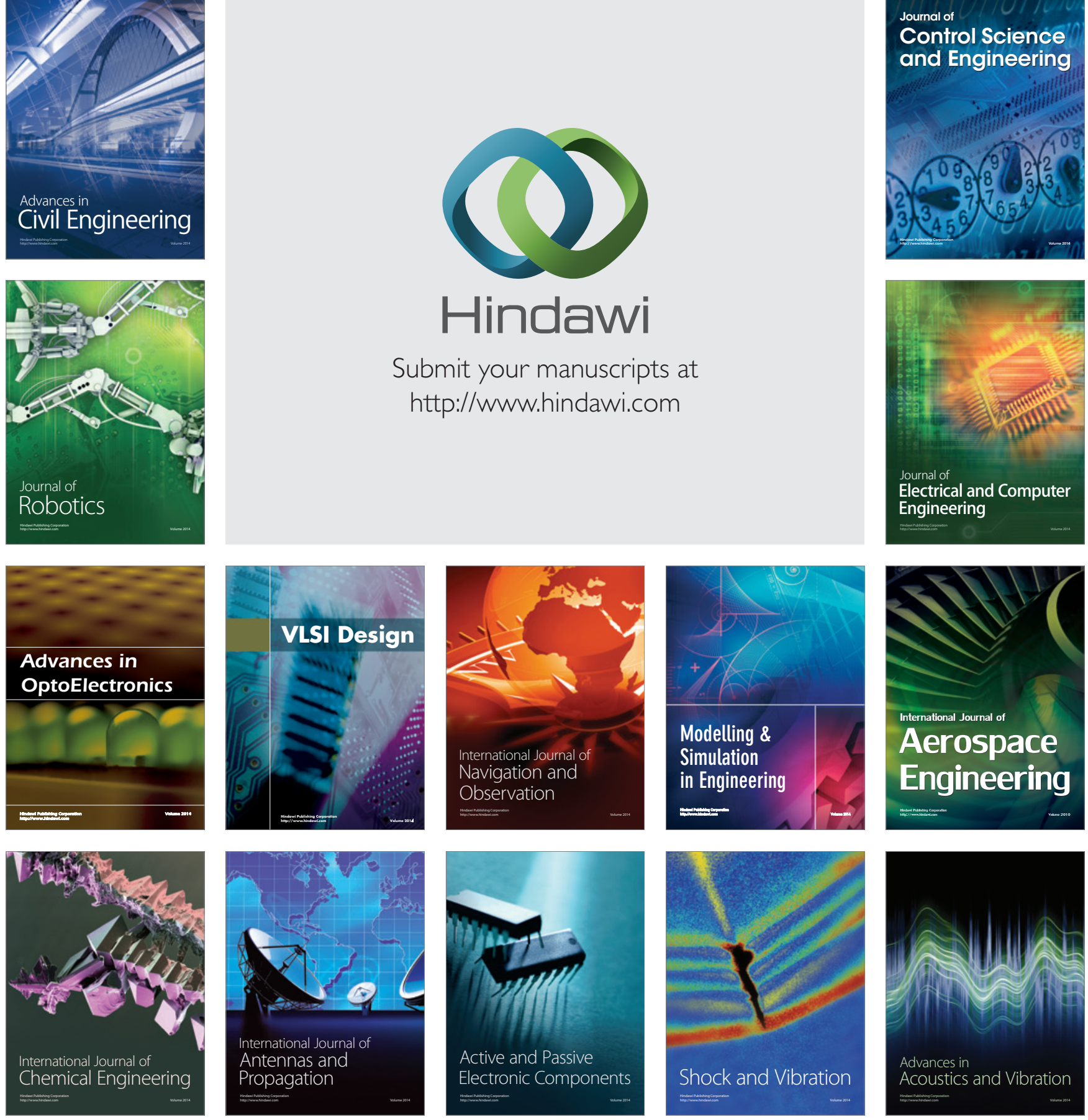